\title{
Segmentation of Exudates from Retinal Image
}

\author{
M. Reema Lovelyn* and Rama Prasath \\ Department of Computing Sciences, MCA, Hindustan Institute of Technology and Sciences, Padur, \\ Chennai -603103, Tamil Nadu, India; Reemalovelyn123@gmail.com, rprasath@hindustanuniv.ac.in
}

\begin{abstract}
Background/Objectives: Exudates are the bright lesions and major cause for blindness. They are the primary signs of diabetic retinopathy. Due to the damage caused in the blood vessels the exudates are formed in the eye and then they form lesions in the retina. They are hard white or yellow in color with different shapes or sizes. They are to be detected at the early stage. Methods/Statistical Analysis: Exudates are the bright lesions. Optic disc is similar to exudates as they have the same intensity as exudates. The edges were detected using the edge detection techniques after this finally the exudates are detected. Findings: This paper deals with the retinal images to detect exudates. Exudates can be detected easily. In some cases where the intensity is low is also detected asexudates so we need to eliminate these unwanted exudates. Work was performed to detect exudates based different techniques. Improvements/Applications: The earlier mentioned techniques used dilated pupils where the exudates as well as the other retinal features were visible. Non-uniform and low contrast images cannot be used for detection. They are reduced using the preprocessing techniques.
\end{abstract}

Keywords: Exudates, Edge Detection, Optic Disc, Preprocessing, Retinal Image

\section{Introduction}

Exudates are mostly caused in working people. The site can be affected due to diabetes which may lead to glaucoma, cataracts and damage of the blood vessels. Poor vision is caused due to exudates. When the disease is detected earlier the progression of exudates can slow down. The damages of the blood vessels are the main cause. They are visible inthe form of clusters, individual streaks, large criminate.

Due to liquid that leaks from the weakened blood vessels the exudates are formed hard. Through the damaged blood vessels the leaked proteins flow from the blood stream to the retina. They are yellowish or white in color with different shapes and sizes. Texture, size, color and shape are the features that are very important to detect exudates. Exudates will not have a uniform shape which makes identification a difficult one. To detect exudates from an image the noise in the image is removed and then the channel with highest intensity is selected. The green channel is used as it gives the between the red lesions and background. Red channel is brighter and has wider range of gray so there is less contrast between lesions and background. There are many techniques to detect the edges like Sobel, Canny, Prewitt, Roberts and Fuzzy.

The edges were detected easily using the Log technique. Techniques of preprocessing are used to reduce the non-uniformity and contrast. Preprocessing techniques are linear filtering, distributed linearization, spatial digital filtering, contrast enhancement, image subtraction. The exact exudates were detected using these techniques. The exudates pixels can be differentiated easily from the pixels that are normal due to their intensity. Standard deviation is used as exudates are highly textured than the non-exudates region and by using standard deviation it is easy to indicate the texture. RGB was used to distinguish the exudates from non-exudates. Many edge pixels are formed due to the small clusters of exudates. The Log edge operation was used to eliminate the edges that are strong and arise from the blood vessels.

The exudates for diabetic retinopathy were detected. The technique that was used is the Fuzzy C-Mean (FCM)

${ }^{*}$ Author for correspondence 
clustering ${ }^{\underline{1}}$. It is used to do the optimal selection of a number ofclusters. Contrast enhancement and pre-processing were employed using featureslike hue, number of edge pixels, intensity and standard deviation.

In development of an automated system which describes to detect abnormalities. To standardize the color and to enhance the contrast image processing was used $^{2}$. Lesions were revealed using segmentation which was followed by neural network for classification of lesions. Few abnormalities are exudates, hemorrhages and microneuysm.

In 2010 presented new method where the exudates that are based on the segmentation that is the markercontrolled watershed, contrast enhancement and average filtering was used to detect the bright objects ${ }^{3}$.

Neural network that was used in gray level images to identify the exudates ${ }^{4}$. To train artificial neural networks the back propagation method was used. Based on the features the image was divided into $30 \times 30$ and $20 \times 20$ pixel of squares. The squares of these gray-scaled images were classified as exudates, hemorrhages, normal, micro aneurysms or blood vessels.

In a system that is used to detect the exudates, hemorrhages and micro aneurysms by the detection of Diabetic Retinopathy automatically ${ }^{5}$. They introduced the Recursive Region Growing Segmentation that was used on $10 \times 10$ windows with selected threshold value to detect the exudates.

\section{Retinal Detection}

\subsection{Exudates}

Exudates are the major cause for blindness. Detection of exudates and the edges was easy. In some cases where the intensity is low is also detected asexudates so we need to eliminate these unwanted exudates. Based on various techniques much work has been performed. Few experiments reported images with larger fields and good quality.

\subsection{Preprocessing}

The intensity play major role to detect the exudates. Intensities with high numbers are the light part of images. The important features are found in the high and low quality images. By using preprocessing techniques such as spatial digital filtering, contrast enhancement, image subtraction, linear filtering we can reduce the contrast and the non-uniform illumination. To enhance local contrast, the adaptive histogram equalization was used. Non-uniform part was detected as exudates due to its less intensity.

The objects of an image can be identified using maxima. An image has maxima in terms of texture and intensity. The pixel that is equal or greater to the threshold is represented as white in color with value 1 and the other pixels are represented as black in color with value 0 . During this process the binary image is formed from the original image. The pixels in white color are represented as the exudates and the rest in black is represented as the background of an image.

\subsection{Edge Enhancement}

Enhances the edge contrast of an image. Identifying sharp edge boundaries. Increasing the image contrast in the area. It can be analog or digital process.

Properties

- Amount - Controls the contrast.

- Radius or aperture - The size of the edges to be detected or enhanced.

- Threshold - To adjust the edge detection mechanism sensitivity.

\subsection{Edge Detection}

Under image segmentation the edge detection is mainly used. Variables that are used in the selection of an edge detection operator consist of Edge structure, orientation and Noise environment $\underline{\underline{6}}$. We used the thresholds based on distribution of intensity value to detect the edges of exudates. Thereholding is used to transformation an input image to an output that is the binary image. Segmentation intensity value is mainly used to detect the edges. After detecting the edges they are fused. Edges can be thickened when not viewed clearly. The detected edges are considered as exudates. Many other places due to less intensity the edges are detected. These edges were removed using preprocessing.

Many edge detection techniques are available to detect the edges in image segmentation. The Laplacian of Gaussian technique computes the Laplacian, gives a double edge image and smoothes the image. The edges are to be located that consists to find the double edges which are between the zero crossings. The Laplacian function is usually made by digital implementation. It is generally 
used to find whether the pixel is on dark or light side of the edge.

\section{Results and Discussion}

In this paper, Figure 1 show the input retinal image which consist of exudates. The RGB image is converted to various channels to detect exudates clearly as shown in Figure $2,3,4$. The $G$ channel that detects the exudates clearly is used for further processing as it gives a better contrast between exudates and background. The colored image is changed to a grayscale image. To remove the noise medium filtering was used. The contrast limited adaptive histogram is used to enhance local contrast. Figure 5 shows the area of the retinal image which was identified as exudates. The edges of the exudates are detected using the Log edge detection technique in Figure 6.

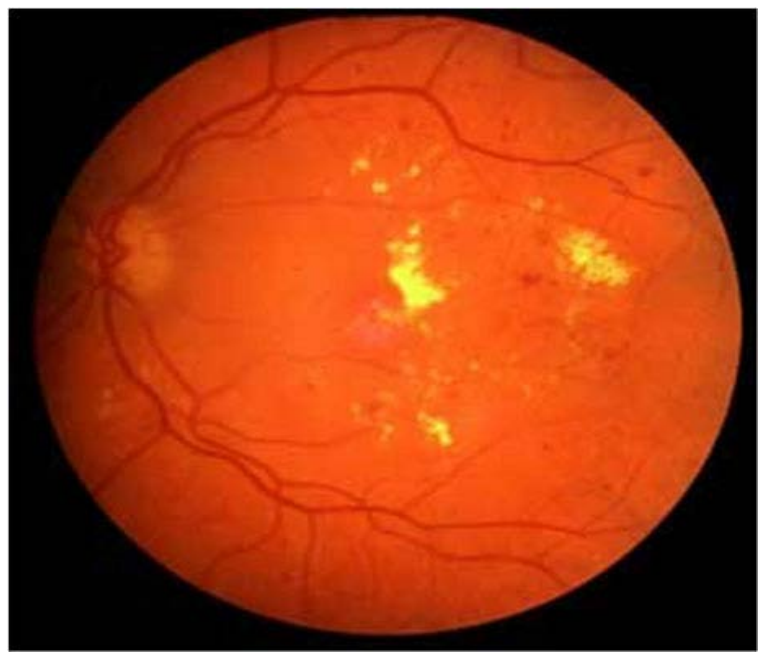

Figure 1. Retinal image.

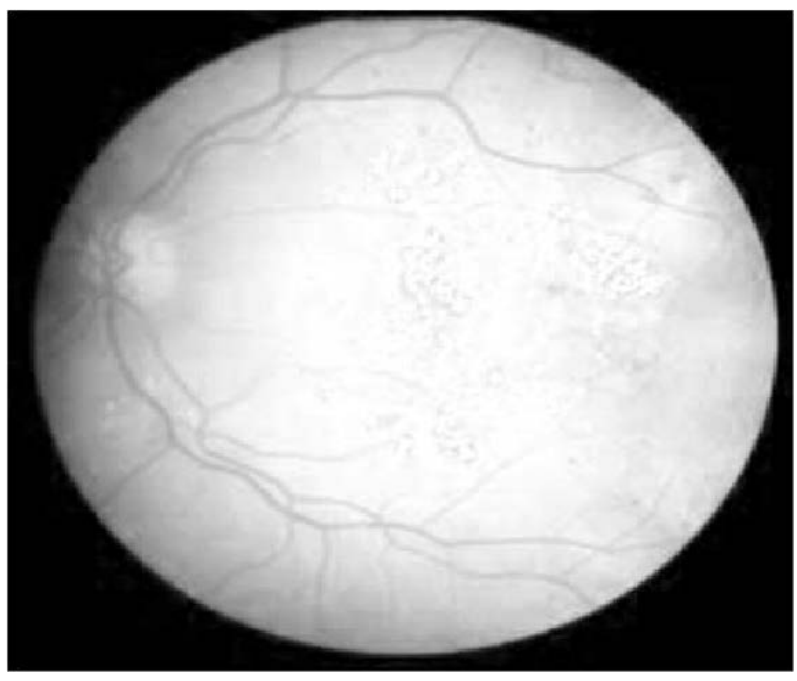

Figure 2. Red channel.

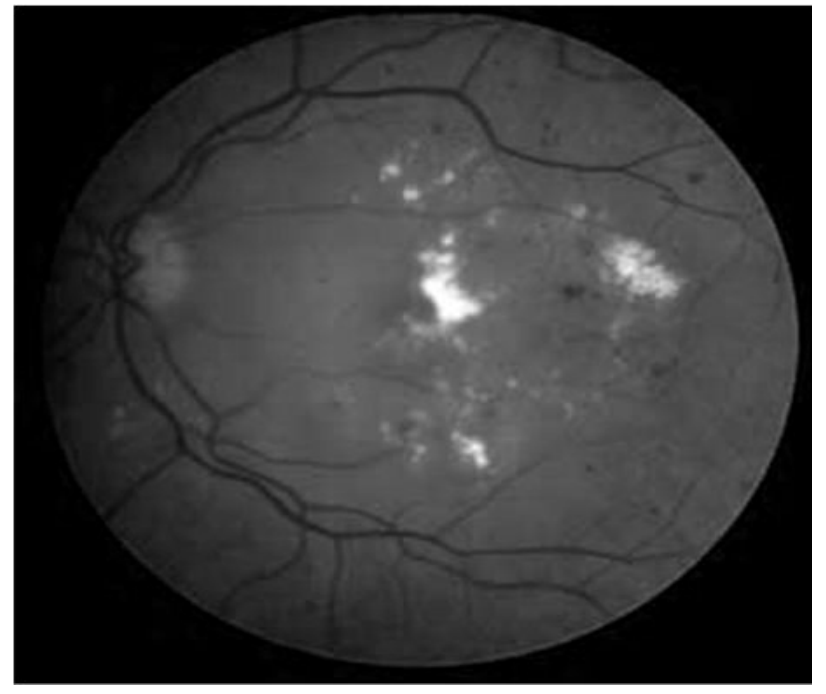

Figure 3. Green channel.

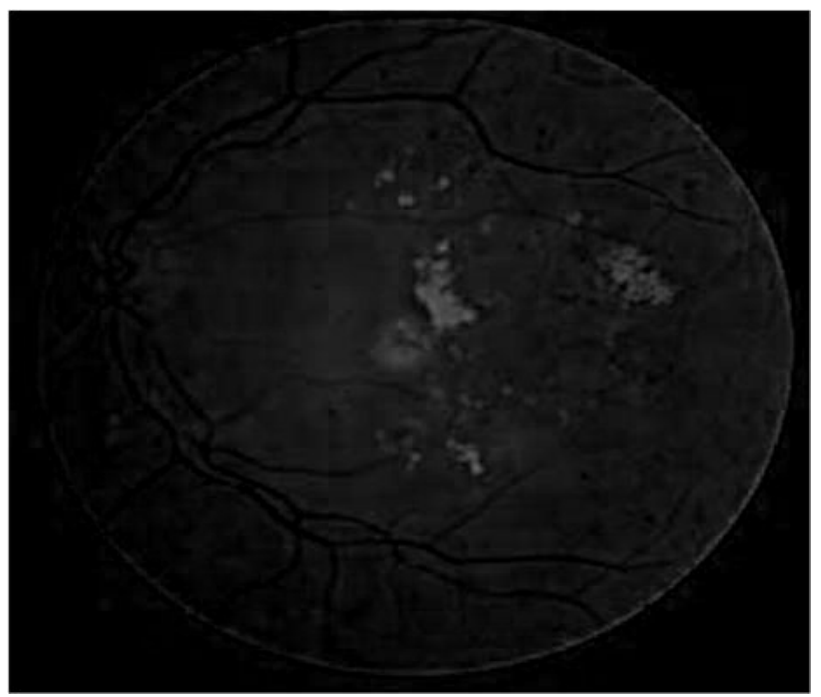

Figure 4. Blue channel.

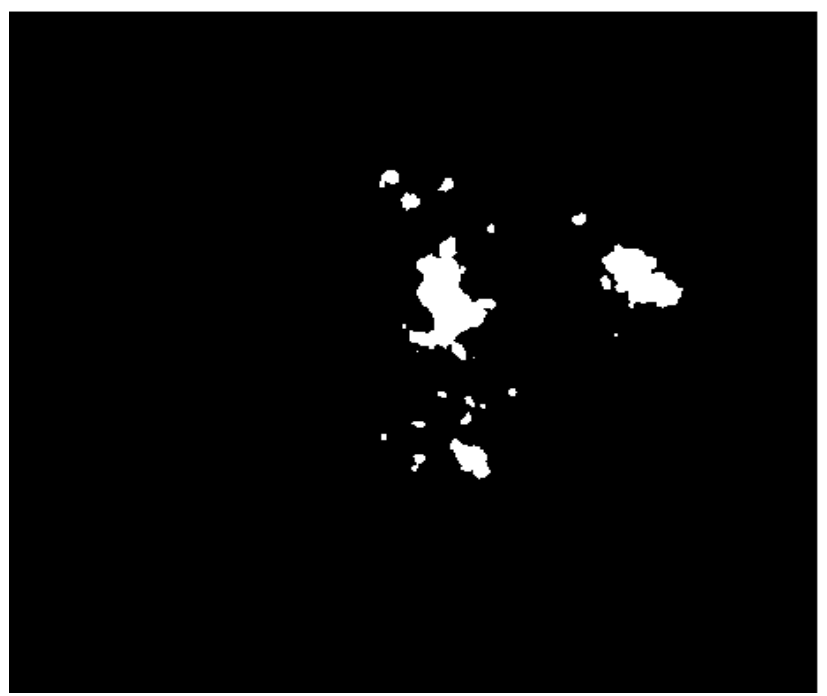

Figure 5. Low intensity area.

Indian Journal of Science and Technology $\mid 3$ 


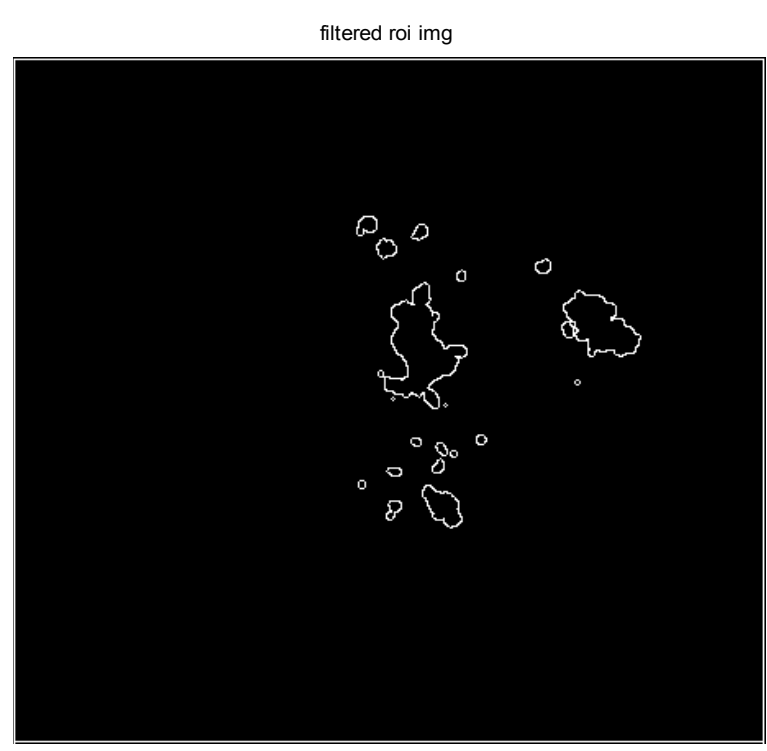

Figure 6. Edges.

\section{Conclusion}

Early detection of exudates can slow down the disease and avert blindness. The Diabetic Retinopathy (DR) is a common complication which leads to blindness. It is a silent disease and is recognized by the patient when there is a change in the retina, that treatment is complicated and nearly impossible. In this paper after the image is read, the image is transformed to the green channel as it gives a better contrast between the exudates and the background. To detect the edges of the exudates the edge detection techniques are used. The contrast and non-uniform illumination are reduced. Edges are fused and then the final exudates are detected.

\section{Acknowledgement}

It is our extreme pleasure to thank our Chancellor Dr. Elizabeth Verghese, Vice-Chancellor Dr. S. Ramachandran, Hindustan University for providing a conductive environment which helped us to pursue our project in a diligent and an efficient manner.
We wish to express our sincere gratitude to Dr.Vasudevan, Dean (Academics), and Dr. D. Venkata Subramanian Dean (School of Computer Sciences), for their encouragement and support to complete this project. We are thankful to Dr. Rajeswari Mukesh, Head of the Department of Computer Applications.We are indebted to our project guide Dr. A. Rama Prasath, Assistant professor, Department of Computer Applications for his stimulating suggestions and encouragement which helped me to coordinate my project especially in writing the paper.Special thanks go to all my staff members and friends who helped me in my coding and gave their valuable suggestions about the project.Last but not least, I would also like to thank God Almighty and my family who were keen in helping me in all ways and to give their blessings.

\section{References}

1. Sopharak A, Uyyanonvara, Barman S, Vongkittirux S, Wongkamchang N. Fine exudates detecting using morphological reconstruction enhancement. International Journal of Applied Biomedical Engineering. 2010; 1(1):1-6.

2. Usher D, Dumsky M, Himaga M, Williamson TH, Nussey SI, Boyce J. Automated detection of DR in digital retinal images. Diabetic Medicine. 2003; 21(1):81-90. Doi: Crossref

3. Phung SL, Bouzerdoum A, Chai D. Skin segmentation using color pixel classification: analysis and comparison. IEEE Transactions on Pattern Analysis and Machine Intelligence. 2005; 27(12):148-54. Crossref

4. Gao W, Yang L, Zhang X, Liu H. Improved sobel edge detection. 3rd International Conference on Computer Science and Information Technology; 2010. p. 67-71.

5. Zhengyao W. Edge detection of digital image. Xi»an: i»an Jiaotong University; 2003.

6. Zheng YY, Rao JL, Wu L. Edge detection methods in digital image processing. The 5th IEEE International Conference on Computer Science and Education; 2010. p. 471-3. Crossref 